\title{
Fast and Simple One-Way High-Dimensional Quantum Key Distribution
}

\author{
Kfir Sulimany ${ }^{1}$, Rom Dudkiewicz ${ }^{2}$, Simcha Korenblit ${ }^{1}$, Hagai S. Eisenberg${ }^{1}$, Yaron Bromberg ${ }^{*}$, \\ and Michael Ben-Or ${ }^{*}$ \\ ${ }^{1}$ Racah Institute of Physics, The Hebrew University of Jerusalem, Jerusalem 91904, Israel \\ ${ }^{2}$ School of Computer Science \& Engineering, The Hebrew University of Jerusalem, Jerusalem, \\ 91904 Israel \\ *Corresponding authors: yaron.bromberg@mail.huji.ac.il, benor@cs.huji.ac.il
}

\begin{abstract}
High-dimensional quantum key distribution (QKD) provides ultimate secure communication with secure key rates that cannot be obtained by QKD protocols with binary encoding. However, so far the proposed protocols required additional experimental resources, thus raising the cost of practical high-dimensional systems and limiting their use. Here, we analyze and demonstrate a novel scheme for fiber-based arbitrary-dimensional QKD, based on the most popular commercial hardware for binary time bins encoding. Quantum state transmission is tested over $40 \mathrm{~km}$ channel length of standard single-mode fiber, exhibiting a two-fold enhancement of the secret key rate in comparison to the binary Coherent One Way (COW) protocol, without introducing any hardware modifications. This work holds a great potential to enhance the performance of already installed QKD systems by software update alone.
\end{abstract}

\section{Introduction}

Quantum key distribution (QKD) is an advanced technology which provides ultimate secure communication by exploiting quantum states of light as information carriers over communication channels $[1,2,3,4$. In the early QKD protocols each bit of the key was encoded using a quantum state belonging to a binary Hilbert space [5, 6]. High-dimensional QKD protocols were introduced more recently, based on preparing a set of states belonging to a d-dimensional Hilbert space, called qudits $7,8,9$, 10. The higher information capacity of qudits allows a higher secure key rate and improves the robustness to noise, leading to higher threshold values of the quantum bit error rate (QBER) 11, 12, 13, 14, 15, 16.

Time-bin encoding of weak coherent laser pulses is the most popular technique for implementing QKD over single-mode fibers $17,18,19,20$. Recent proposals and demonstrations of high-dimensional temporal encoding showed a significant key rate improvement $21,22,23,24$, 25, 26, 27, 28, 29]. In particular, a record-breaking key rate of $26.2 \mathrm{Mbit} / \mathrm{s}$ was achieved with a four-dimensional time-bin protocol that is robust against the most general (coherent) attacks 21.

Implementation of high-dimensional QKD protocols in commercial systems is still held back, since present high-dimensional schemes require significantly higher experimental resources. The large experimental overhead results from the fact that high-dimensional encoding not only increases the channel capacity, but it also increases the amount of information that Eve can extract. Most QKD protocols limit the amount of information accessible to Eve by projecting the quantum states at the receiver's end on unbiased bases. While the projection in binary schemes is usually implemented with a single interferometer followed by a single photon detector (SPD), most d-dimensional schemes require $O(d)$ imbalanced interferometers and $O(d)$ SPDs $21,22,23$. Thus, to date, all high-dimensional QKD systems implementations required complex and expensive systems that are impractical for commercial applications.

In this work we present a different approach for highdimensional QKD with time-bin encoding, which can be implemented using a standard commercial QKD system without any hardware modifications. Instead, we show that Eve's information can be bounded by simply randomizing the time-bins order. We further analyze the security and expected secure key rate for optimized Eve's strategy. Finally, we experimentally demonstrate a 32 dimensional protocol over a $40 \mathrm{~km}$ long fiber using only two single-photon detectors and one interferometer at the receiver end. We demonstrate the improved performance of our protocol in comparison to the binary COW protocol using the same experimental setup, and show more than a two-fold increase in the asymptotically secure key rate. 


\section{Protocol Scheme}

Our high-dimensional protocol is based on the coherent one-way (COW) QKD protocol, where the bit string is encoded in the time of arrival of weak coherent laser pulses and the channel disturbance is monitored by measuring the visibility of the interference between neighbouring pulses $[30]$. That is, bits 0 and 1 are sent using $|\alpha\rangle|0\rangle$ and $|0\rangle|\alpha\rangle$, respectively, where $|0\rangle$ is the vacuum and $|\alpha\rangle$ is a coherent state. On Bob's side, he simply recovers the bit value by measuring the arrival time of the laser pulse. To detect attacks, a small fraction of the pulses splits to a monitoring line by a fiber beam splitter. In the monitoring line Bob checks for phase coherence between any two successive laser pulses by using an imbalanced interferometer and one single photon detector.

Although the the COW protocol is very popular due to the simplicity of its implementation 31, 32, 33, 34 17, 35, 36, 37, 38, in recent years there is a development in the analysis of the secure key rate. An unconditional security proof of COW-QKD is crucial but still elusive. General security bounds against individual attacks and upper bounds for the error rates against coherent attacks have been derived for the COW protocol [39]. Considering the collective attacks the key rate depends linearly on transmittance. Later, lower bounds on the key generation rate in a finite-size key scenario against general attacks have been obtained 40]. In the security proof against general attacks the secure key rate appears to scale quadratically with transmittance. However, so far all COW-QKD experiments $31,32,33,34,17,35,36$, 37. 38], still employ the original security proof [39].

More recently, zero-error attacks, an eavesdrop without breaking the coherence between adjacent nonvacuum pulses, have also been studied for the COW protocol [41, 42, 43. Consequently, COW with key rate scaling linearly with transmittance is totally insecure under the zero-error attack. However, these attacks restrict the secure key rate scale quandratically with the transmittance. Lately, a secure key rate which scales with $0.007 \%$ of the bound that found in $41,42,43$, that scales quadratically with the transcendence, has been found by more precisely estimating the amount of leaked information.

Our extension to a high dimension is based on a more efficient utilization of the quantum bit duration relative to the deadtime of the detector, since the deadtime limits the number of bits that can be received per second. We encode the qudits of the raw key by a sequence of $d$ time slots, where in each sequence only one time slot is populated and the rest are empty. We group $n$ sequences to a block and apply a random permutation on each block to create a permuted key block. We then convert the block to sequence of occupied and non-occupied pulses. Formally, let $q_{0}, \ldots, q_{n-1} \in\{1 \ldots d\}$ be the raw key Alice wants to transmit. Alice chooses a random permutation $\sigma$ of $\{1 \ldots d \cdot n\}$ and over the next $d \cdot n$ time bins sends $|\alpha\rangle$ at time slot $t$ if $t=\sigma\left(d \cdot i+q_{i}\right)$ for some $i$ in $\{0 \ldots n-1\}$ and $|0\rangle$ otherwise as illustrated in Fig. 1. After Bob measures the pulse sequence, Alice transmits $\sigma$ over the classical channel. When Bob detects a click at time $t$, he calculates $\sigma^{-1}(t)=i \cdot d+j$ for $i \in\{0 \ldots n-1\}$ and $j \in\{1 \ldots d\}$ meaning the value passed in the $i$ 'th qudit was $j$. Bob transmits back to Alice which qudits he received, such that the information now is mutual up to error correction.

The random permutation plays a key role in our protocol, as it guarantees that two successive occupied pulses can originate anywhere in the raw key block. This scrambling allows us to bound Eve's information and extract a higher secure key rate, even though our monitoring interferometer probes the coherence of consecutive pulses only.

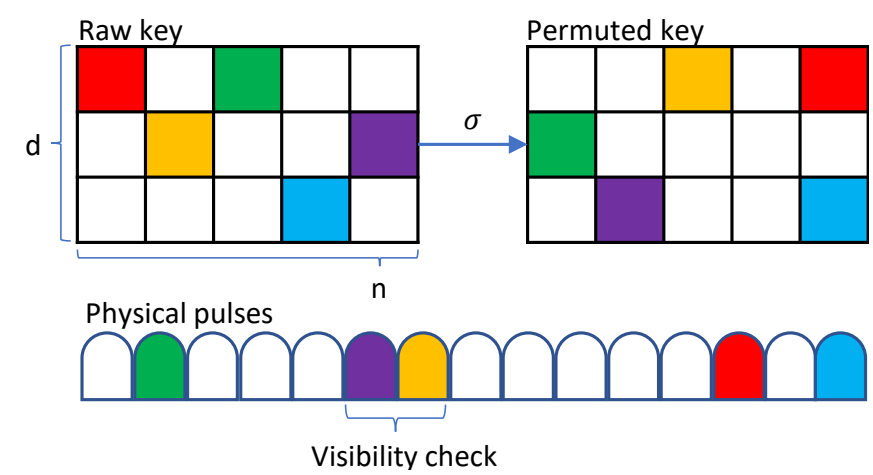

Figure 1: Protocol scheme. Alice produces a secret key consisting of a block of $n d$ random numbers where $d$ is the dimensional encoding and $\mathrm{n}$ is an integer. She permutes the block with a random secret permutation to get a scrambled block, and transmits accordingly a series of occupied and empty pulses. Therefore, a pair of sequentially occupied pulses can originate from any two time slots in the raw key block, an important feature of the protocol that is essential for the security proof.

\section{Security Analysis}

We now turn to explain the protocol in detail, present the encoding states and Eve's strategy, and compute the Holevo information and the number of secure bits per photon. Since our protocol is a variation on the binary COW protocol, we follow the analysis tools presented in 39 .

In principle, Eve can act on blocks of multiple pulses each time. However, since Alice and Bob mix arbitrary pulses in order to define key bits, Eve cannot find a natural way to apply an attack on multiple pulses 39 . We can thus focus on Eve's action on a single time slot, which can be defined by a linear transformation describing the 
action on an non-occupied and occupied time-slots:

$$
\begin{gathered}
|0\rangle_{A}|\varepsilon\rangle_{E} \rightarrow|0\rangle_{B}\left|v_{0}\right\rangle_{E}+\sqrt{Q \mu t}|1\rangle_{B}\left|p_{0}\right\rangle_{E} \\
|\sqrt{\mu}\rangle_{A}|\varepsilon\rangle_{E} \rightarrow|0\rangle_{B}\left|v_{\mu}\right\rangle_{E}+\sqrt{(1-(d-1) Q) \mu t}|1\rangle_{B}\left|p_{\mu}\right\rangle_{E},
\end{gathered}
$$

where $\mathrm{Q}$ is the quantum bit error rate (QBER) i.e. the probability that Bob receives a wrong bit value. $\mu$ and $t$ are the pulse occupation and the link transmission, respectively. The amplitudes of the states guarantee that Eve's attack does not increase the QBER.

The loss of coherence is monitored analyzing the detection events in the monitoring line. The phase between the two arms in the monitoring line is chosen so that two consecutive non-empty pulses sent by Alice will interfere destructively in one output port and constructively in the other port. The loss of coherence by Eve's attack can then by quantified by the visibility:

$$
V=\frac{P\left(D_{c}\right)-P\left(D_{d}\right)}{P\left(D_{c}\right)+P\left(D_{d}\right)},
$$

where $P\left(D_{c}\right)$ and $P\left(D_{d}\right)$ are the probabilities to measure a photon at the constructive and destructive ports, respectively. Since Eve's attack should keep the visibility unchanged, we can derive a constraint on Eve's action on two consecutive pulses sent by Alice $|\sqrt{\mu}\rangle|\sqrt{\mu}\rangle$. Assuming $\mu t \ll 1$, Eve's action is given by:

$$
\begin{gathered}
|\sqrt{\mu}, \sqrt{\mu}\rangle_{A}|\varepsilon, \varepsilon\rangle_{E} \rightarrow|0,0\rangle_{B}\left|v_{\mu}, v_{\mu}\right\rangle_{E} \\
+\sqrt{(1-(d-1) Q) \mu t}\left[|1,0\rangle_{B}\left|p_{\mu}, v_{\mu}\right\rangle_{E}\right. \\
\left.+|0,1\rangle_{B}\left|v_{\mu}, p_{\mu}\right\rangle_{E}\right],
\end{gathered}
$$

The visibility constraint then yields (see Supplementary Information):

$$
V=\left|\left\langle p_{\mu} \mid v_{\mu}\right\rangle\right|^{2} .
$$

The last constraint on Eve's transformation is that it must be unitary. Therefore:

$$
\left\langle v_{0} \mid v_{\mu}\right\rangle=e^{-\mu / 2}
$$

Our security analysis is therefore based on three constraints imposed on Eve's action: i) It must retain the QBER (eq. 1), ii) it must keep the visibility (eq. 5), and iii) it must be unitary (eq. 6).

To compute the amount of information that can be extracted by Eve, quantified by the Holevo information, we first need to analyze her action on a qudit with occupation $\mu^{(i)}$ in the $i^{t h}$ time slot. Neglecting all multiple photon terms, Eve's action can be presented by:

$$
\begin{gathered}
\left|0, \ldots, 0, \sqrt{\mu}^{(i)}, 0, \ldots, 0\right\rangle_{A}|\varepsilon, \ldots, \varepsilon\rangle_{E} \rightarrow \\
|0, \ldots, 0\rangle_{B} \otimes\left|V_{i}\right\rangle \\
+\sqrt{(1-(d-1) Q) \mu t}\left|0, \ldots, 0,1^{(i)}, 0 \ldots, 0\right\rangle_{B} \otimes\left|C_{i}\right\rangle \\
+\sum_{k=1 \ldots d, k \neq i} \sqrt{Q \mu t}\left|0, \ldots, 0,1^{(k)}, 0 \ldots, 0\right\rangle_{B}\left|W_{i, k}\right\rangle,
\end{gathered}
$$

where $\left|V_{i}\right\rangle=\left|v_{0}, \ldots, v_{0}, v_{\mu}^{(i)}, v_{0}, \ldots, v_{0}\right\rangle_{E}$ is Eve's state representing the case where she sends a vacuum state at time slot $i,\left|C_{i}\right\rangle=\left|v_{0}, \ldots, v_{0}, p_{\mu}^{(i)}, v_{0}, \ldots, v_{0}\right\rangle_{E}$ is Eve's state representing the case where she sends to Bob a photon at the right time slot $i$, and $\left|W_{i, k}\right\rangle=$ $\left|v_{0}, \ldots, v_{0}, p_{0}^{(k)}, v_{0}, \ldots, v_{0}, v_{\mu}^{(i)}, v_{0}, \ldots, v_{0}\right\rangle_{E}$ is Eve's state representing the case where she sends to Bob a photon at the wrong time slot $k$.

Next, we compute the density matrices of Eve's subsystem, conditioned by the event where Alice sends a pulse at time slot $i$ and Bob detects a photon at some arbitrary time slot:

$$
\begin{gathered}
\rho_{E}^{A=i}=(1-(d-1) Q)\left|C_{i}\right\rangle\left\langle C_{i}\right| \\
+\sum_{k=1 \ldots d, k \neq i} Q\left|W_{i, k}\right\rangle\left\langle W_{i, k}\right|
\end{gathered}
$$

Similarly, the density matrix of Eve's subsystem conditioned by the event where Bob detects a pulse at time slot $i$ and Alice sent the pulse at an arbitrary time slot:

$$
\begin{gathered}
\rho_{E}^{B=i}=(1-(d-1) Q)\left|C_{i}\right\rangle\left\langle C_{i}\right| \\
+\sum_{k=1 \ldots d, k \neq i} Q\left|W_{k, i}\right\rangle\left\langle W_{k, i}\right|
\end{gathered}
$$

The Holevo bounds on Alice-Eve channel $\chi_{A E}$ and on Bob-Eve channel $\chi_{B E}$, are defined by:

$$
\begin{aligned}
& \chi_{A E}=S\left(\sum_{i=1}^{d} \frac{1}{d} \rho_{E}^{A=i}\right)-\sum_{i=1}^{d} \frac{1}{d} S\left(\rho_{E}^{A=i}\right) \\
& \chi_{B E}=S\left(\sum_{i=1}^{d} \frac{1}{d} \rho_{E}^{B=i}\right)-\sum_{i=1}^{d} \frac{1}{d} S\left(\rho_{E}^{B=i}\right)
\end{aligned}
$$

where $S(\rho)=-\operatorname{Tr}\left(\rho \log _{2} \rho\right)$ is the von Neumann entropy of $\rho$. The maximal information Eve can extract is bounded by the maximum of these two quantities. Direct computation of eq. 10 shows that $\chi_{B E}>\chi_{A E}$ (see Supplementary Information), hence from this point on we will focus on analyzing $\chi_{B E}$.

Eve has no constraints over $\left|p_{0}\right\rangle$ as it does not affect the three constraints imposed by eqs.(1),(5) and (6). Thus, in order to maximize her information she can choose $\left|p_{0}\right\rangle$ that is orthogonal to all other vectors $\left|v_{0}\right\rangle,\left|v_{\mu}\right\rangle,\left|p_{\mu}\right\rangle$. Conveniently, we can then separate the trace of the above matrices to a trace of density matrices that contain only one $\left|p_{0}\right\rangle$ in time slot $i$ for $i=1 . . d$, and 
a trace of matrices that do not contain $\left|p_{0}\right\rangle$, yielding (see Supplementary Information):

$$
\begin{gathered}
\chi_{B E}= \\
\sum_{k=1}^{d}\left[S\left(\sum_{i=1}^{d-1} \frac{Q}{d}\left|W_{i}^{\prime}\right\rangle\left\langle W_{i}^{\prime}\right|\right)-\frac{1}{d} S\left(\sum_{i=1}^{d-1} Q\left|W_{i}^{\prime}\right\rangle\left\langle W_{i}^{\prime}\right|\right)\right] \\
+S\left(\sum_{i=1}^{d-1} \frac{1-(d-1) Q}{d}\left|C_{i}\right\rangle\left\langle C_{i}\right|\right) \\
-\frac{1}{d} \sum_{i=1}^{d} S\left((1-(d-1) Q)\left|C_{i}\right\rangle\left\langle C_{i}\right|\right)
\end{gathered}
$$

where we define $\left|W_{i}^{\prime}\right\rangle=\overbrace{\left|v_{0}, \ldots, v_{0}, v_{\mu}^{(i)}, v_{0}, \ldots, v_{0}\right\rangle}^{d-1 \text { terms }}$, such that $\left|W_{i}^{\prime}\right\rangle \otimes\left|p_{0}\right\rangle=\left|W_{i, d}\right\rangle$ and $\left|W_{i, j}\right\rangle$ are equivalent up to reordering the order of the time slots.

After diagonalization of the density matrix and applying the traces, we obtain the following expression for the Holevo information (See Supplementary Information):

$$
\begin{gathered}
\chi_{B E}=Q(d-1) \log _{2}(d) \\
+S\left(\frac{1-(d-1) Q}{d}\left((d-1)\left|\left\langle v_{0} \mid p_{\mu}\right\rangle\right|^{2}+1\right)\right) \\
+(d-1) S\left(\frac{1-(d-1) Q}{d}\left(1-\left|\left\langle v_{0} \mid p_{\mu}\right\rangle\right|^{2}\right)\right) \\
-S(1-(d-1) Q)
\end{gathered}
$$

To maximize eq. 12 , we can minimize $\left\langle v_{0} \mid p_{\mu}\right\rangle$ under the constraints imposed by eq. 5 and eq. 6. Using a parametric representation of $\left|v_{0}\right\rangle,\left|p_{u}\right\rangle$ and $\left|v_{u}\right\rangle$ in 3$\mathrm{D}$ space, we find that the maximal information Eve can extract $\max \left\{\chi_{B E}\right\}$ is obtained for:

$$
\left\langle v_{0} \mid p_{\mu}\right\rangle=e^{-\mu / 2} \sqrt{V}-\sqrt{1-e^{-\mu}} \sqrt{1-V}
$$

An upper bound on the secure key fraction can now be computed, using the bound 39].

$$
\begin{gathered}
I_{A B}=\log _{2}(d)+(d-1) Q \log _{2}(Q)+ \\
(1-(d-1) Q) \log _{2}(1-(d-1) Q) \\
-\max \left\{\chi_{B E}\right\}
\end{gathered}
$$

\section{Experimental Implementation}

The important feature of our high-dimensional protocol is that it is implemented in a standard binary COW system as depicted in Fig. 2 without any hardware changes. The system consists of a transmitter (Alice) and a receiver (Bob). The transmitter sends a train of weak coherent pulses that are prepared from a continuous wave (CW) laser emitting at $\lambda=1550 \mathrm{~nm}$, by an intensity modulator (IM) running at $500 \mathrm{MHz}$. Before leaving the transmitter, the pulses are attenuated to reach single photon level using a variable optical attenuator (VOA). To generate $200 p s$ long pulses with random occupations of $\tau=2 n s$ long time-bins we use field programmable gate array (FPGA). Synchronization is achieved over the 40 $\mathrm{km}$ fiber channel using the White Rabbit protocol 44]. To interfere consecutive pulses at the receiver's end, a fiber unbalanced Michelson interferometer is installed, where we use Faraday mirrors to compensate for random polarization drifts in the fiber interferometer (Fig. $2 \mathrm{~b}$ ). We use single-photon avalanche detectors (SPADs) with $20 \%$ detection efficiency and 400ps timing resolution. The detectors' dead time is $4 \mu s$, limiting the maximal raw key rate to $250 \mathrm{kHz}$.

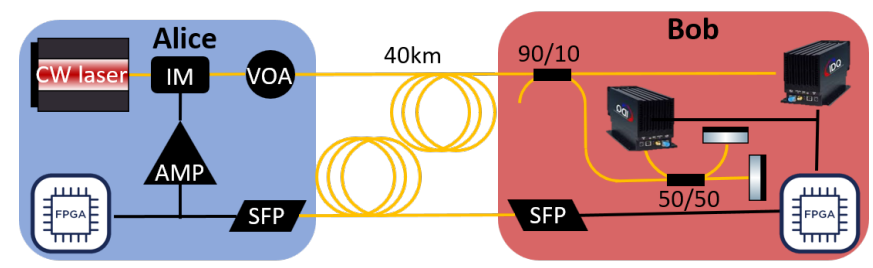

Figure 2: Experimental setup for comparing arbitrary-dimensional QKD schemes. Alice's transmitter (left) consists of a continuous wave (CW) laser at $\lambda=1550 \mathrm{~nm}$ that is modulated using an electro-optic intensity modulator (IM) running at $500 \mathrm{M} \mathrm{Hz}$. The pulses are passed through a variable optical attenuator (VOA) that regulates the mean photon number per pulse. The weak coherent pulses are delivered to Bob's end through a $40 \mathrm{~km}$ long single mode fiber (SMF-28). Bob's receiver (right) consists of an asymmetric beamsplitter, which provides a passive choice of the measurement basis; $90 \%$ of the photons travel directly to the data detector, and $10 \%$ pass through an unbalanced interferometer and detected by the monitor detector. We lock the laser's wavelength to the interferometer so that the monitor detector always measures the dark port of the interferometer. The interference visibility is estimated by registering the detection events due to the interfering and non-interfering events. In addition to the $40 \mathrm{~km}$ long quantum channel that delivers the weak coherent pulses, we use a separate $40 \mathrm{~km}$ SMF-28 fiber for all classical communication between Alice and Bob and to distribute an optical clock signal between them based on he White Rabbit protocol 44]. State preparation and sifting is run by two fieldprogrammable gate arrays (FPGA) at Alice's and Bob's ends. 

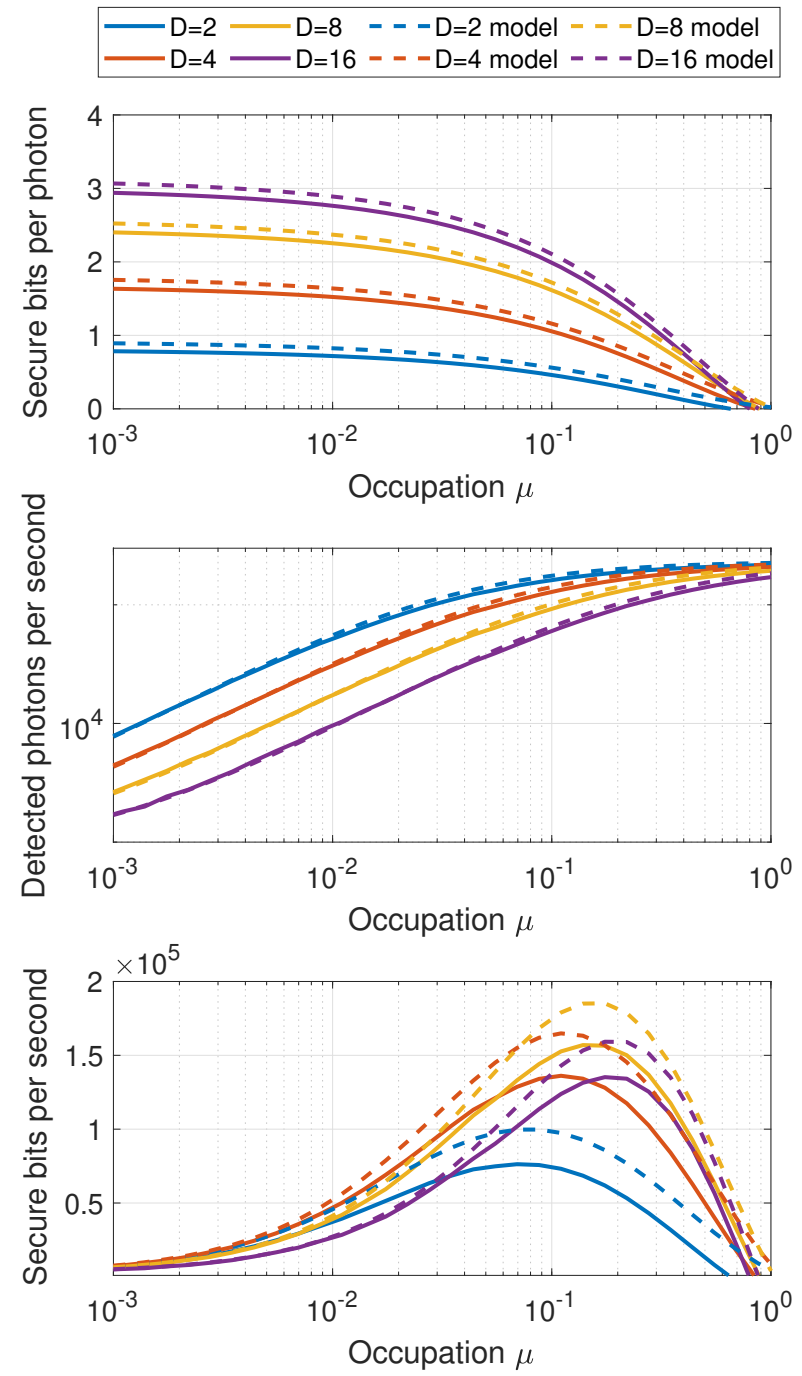

Figure 3: Key rates for different dimensions. a) Number of secure bits per photon (solid) for our system and for the model system (dashed). Increasing the pulse occupation weakens the constraints on Eve and therefore increases the information she can obtain, yielding a lower nuber of secure bits per photon. Higher dimensional encoding allows a higher number of secure bits per photon. b) Number of raw bits per photon in our system (solid) and the calculated raw bits per second for the model system. c) Secure bits per second is the multiplication of the raw bits per photon by photons per second. The optimum is achieved at $d=8$, where the number of secure bits per second increases by 2.04 for our system, and by 1.89 for the model.

We analyze the performance of the protocol for different dimension sizes, and compare the experimental results obtained with our QKD system with the predictions of a cleaner theoretical model. We first calculate the number of secure bits per photon as a function of the pulse occupation for different dimensions, as presented in Fig. 3a. Solid lines present the number of secure bits per photon for our system, based on the measured QBER and visibility for each dimensional encoding size and eq. 14 The dashed lines present the calculated secure bits per photon for the theoretical model, where we assumed visibility of $99 \%$ and a QBER of $0.4 \%$ per time slot. Here we also assumed the QBER scales linearly with the dimension size. The main source for such linear scaling is the finite extinction ratio of the intensity modulator, typically on the order of 0.01. In the limit of low occupation, the deterors' dark counts may become the dominate source for linear scaling of the QBER with the dimension size.

As appears in Fig. 3 a, higher dimensional encoding allows higher secure bits per photon. At the same time, increasing the pulse occupation weakens the constraints on Eve and therefore increases the information she can obtain, decreasing the number of secret bits per photon. In Fig. 3b we present the number of detected photons per second versus the pulse occupation, for different dimensions. The solid lines present the measured number of detected photons per second, and the dashed lines present the calculated detection rate (see Supplementary Information): $\frac{1}{T+\tau \frac{D}{\xi \mu}}$. The number of raw bits per photon increase linearly up to occupation of around 0.05 where the detector starts to saturate. In Fig. 35 we present the secure bits per second, obtained by multiplying the raw bits per photon by the number of detected photons per second. It is evident that an optimal secure bit rate is achieved for $d=8$, resulting in more than a two-fold increase in the secure bits rate for both the experimental data and for the theoretical model.

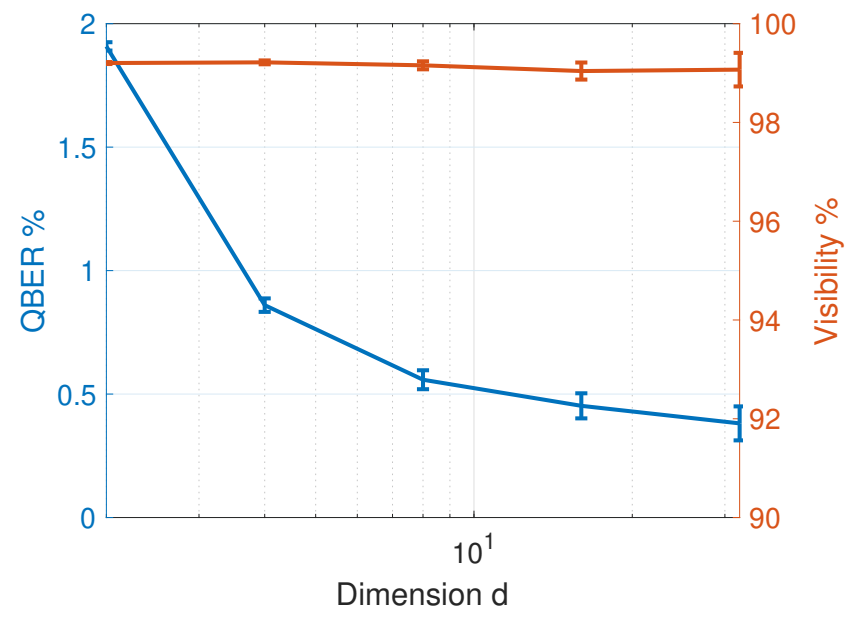

Figure 4: QBER and visibility as a function of the protocol's dimension. The QBER per encoding time bin decreases with the dimension size, due to electronic ringing common in high-rate modulation systems. The measured visibility is insensitive to the dimension size. Error bars are calculated assuming shot-noise limited detection. 
While the experimental results and theoretical model exhibit similar trends, the model fails to capture the exact secure bit rate, due to the oversimplification of the model that assume linear scaling of the QBER with the dimension size. In practice, one of the main noise sources in fast modulation transmitters is cross-talk between consecutive pulses, due to electronic ringing. Since higher dimensions result in longer average times between consecutive pulses, the sensitivity to cross-talk between consecutive pulses decreases with the dimension size. In our system we experimentally observed that the QBER per encoding time bin decreases as the dimension is increased (Fig. 4), yielding higher secure bit rates than the model's prediction.

\section{Discussion}

To asses the resilience of our protocol to noise, we calculate the secure key rate per photon versus the bit error rate for different dimensional encoding sizes as presented in Fig. 5. For $d=2$ we were able to extract secure key rate up to QBER of $15.4 \%$, while for $d=16$ the maximal QBER the protocol could tolerate reduced to $2.7 \%$. This is caused by the linear scaling of the error rate with the dimension due to the leakage of the modulator and the dark counts. Our protocol is therefore not optimal for increasing the communication distances. Fortunately, however, our protocol is useful in many commercially relevant scenarios, since in realistic systems the typical error rate is lower than a few percent 45 .

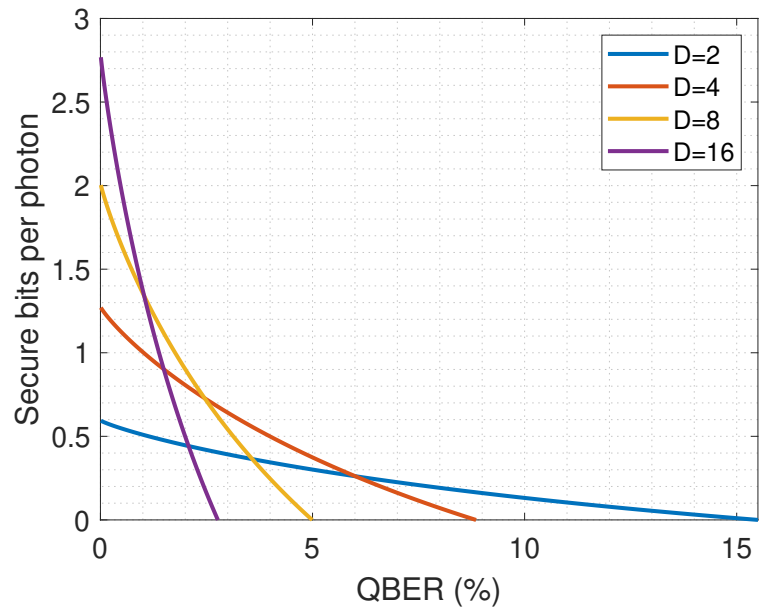

Figure 5: Secret key rate per photon as function of the bit error rate for dimensions $d=2,4,8,16$. The binary case is the most robust to noise. Increasing the dimension decreases the maximal QBER that allows positive secure key rate per photon.

So far we focused on the most popular and cost effective commercial design based on standard single photon
APDs and showed a significant enhancement of the secure bit rate. A similar analysis shows an improvement also for high-end QKD systems based on superconducting nanowire detectors. For example, considering 10 $\mathrm{ns}$ dead time and modulation rates as high as 10Gbps, the secure bit rate at dimension $d=8$ may increase by a factor of 2 compared to standard binary COW encoding.

\section{Conclusion}

In conclusion, we presented a new arbitrary highdimensional QKD protocol, supported by a security proof, which has the advantage of requiring only standard binary QKD hardware. The protocol was experimentally tested with a standard binary encoding system and its performance was tested for several dimension sizes. We demonstrated more than a two-fold enhancement of the secure key rate in the saturation regime of APD detectors. Our demonstration proves that highdimensional quantum systems allow a significant improvement in the key generation rate as compared with the binary-encoding case. At the same time, no additional hardware is required to fully implement the protocol in standard two-dimensional systems. Moreover, the protocol is not limited to time-bins encoding. For example, generalizing our protocol to the spatial domain using non-overlapping Gaussian beams and a single Michelson interferometer, may offer new opportunities for implementing high-dimensional QKD with spatial encoding [46, 47, 48, 49].

Acknowledgements This research was supported by the United States-Israel Binational Science Foundation (BSF) (Grant No. 2017694), and by the Israel Science Foundation (ISF) grant No. 2137/19. KS and YB acknowledge the support of the Israeli Council for Higher Education, the Israel National Quantum Initiative (INQI) and the Zuckerman STEM Leadership Program.

\section{References}

[1] Charles H Bennett and David P DiVincenzo. "Quantum information and computation". In: nature 404.6775 (2000), pp. 247-255.

[2] Nicolas Gisin et al. "Quantum cryptography". In: Reviews of modern physics 74.1 (2002), p. 145.

[3] Stefano Pirandola et al. "Advances in quantum cryptography". In: Advances in Optics and Photonics 12.4 (2020), pp. 1012-1236.

[4] Nurul T Islam. High-rate, high-dimensional quantum key distribution systems. Springer, 2018.

[5] Charles H Bennett and Gilles Brassard. Proceedings of the ieee international conference on computers, systems and signal processing. 1984. 
[6] Artur K Ekert. "Quantum cryptography based on Bell's theorem". In: Physical review letters 67.6 (1991), p. 661.

[7] Helle Bechmann-Pasquinucci and Wolfgang Tittel. "Quantum cryptography using larger alphabets". In: Physical Review A 61.6 (2000), p. 062308.

[8] Nicolas J Cerf et al. "Security of quantum key distribution using d-level systems". In: Physical review letters 88.12 (2002), p. 127902.

[9] Lana Sheridan and Valerio Scarani. "Security proof for quantum key distribution using qudit systems". In: Physical Review A 82.3 (2010), p. 030301.

[10] Jonathan Leach et al. "Secure information capacity of photons entangled in many dimensions". In: Physical Review A 85.6 (2012), p. 060304.

[11] Daniele Cozzolino et al. "High-Dimensional Quantum Communication: Benefits, Progress, and Future Challenges". In: Advanced Quantum Technologies 2.12 (2019), p. 1900038.

[12] Jacob Mower et al. "High-dimensional quantum key distribution using dispersive optics". In: Physical Review A 87.6 (2013), p. 062322.

[13] Tian Zhong et al. "Photon-efficient quantum key distribution using time-energy entanglement with high-dimensional encoding". In: New Journal of Physics 17.2 (2015), p. 022002.

[14] Darius Bunandar et al. "Practical highdimensional quantum key distribution with decoy states". In: Physical Review A 91.2 (2015), p. 022336 .

[15] Catherine Lee et al. "High-rate field demonstration of large-alphabet quantum key distribution". In: arXiv preprint arXiv:1611.01139 (2016).

[16] Catherine Lee et al. "Large-alphabet encoding for higher-rate quantum key distribution". In: Optics express 27.13 (2019), pp. 17539-17549.

[17] Boris Korzh et al. "Provably secure and practical quantum key distribution over $307 \mathrm{~km}$ of optical fibre". In: Nature Photonics 9.3 (2015), pp. 163168.

[18] Alberto Boaron et al. "Secure quantum key distribution over $421 \mathrm{~km}$ of optical fiber". In: Physical review letters 121.19 (2018), p. 190502.

[19] Beatrice Da Lio et al. "Experimental demonstration of the DPTS QKD protocol over a $170 \mathrm{~km}$ fiber link". In: Applied Physics Letters 114.1 (2019), p. 011101.

[20] Frédéric Bouchard et al. "Quantum communication with ultrafast time-bin qubits". In: arXiv preprint arXiv:2106.09833 (2021).

[21] Nurul T Islam et al. "Provably secure and high-rate quantum key distribution with time-bin qudits". In: Science advances 3.11 (2017), e1701491.
[22] Nurul T Islam et al. "Scalable high-rate, highdimensional time-bin encoding quantum key distribution". In: Quantum Science and Technology 4.3 (2019), p. 035008.

[23] Ilaria Vagniluca et al. "Efficient time-bin encoding for practical high-dimensional quantum key distribution". In: Physical Review Applied 14.1 (2020), p. 014051.

[24] Kai Wang et al. "Round-robin differential phasetime-shifting protocol for quantum key distribution: theory and experiment". In: Physical Review Applied 15.4 (2021), p. 044017.

[25] Mirdit Doda et al. "Quantum key distribution overcoming extreme noise: simultaneous subspace coding using high-dimensional entanglement". In: Physical Review Applied 15.3 (2021), p. 034003.

[26] Michał Jachura et al. "Photon-efficient quantum key distribution using multiqubit time-bin encoding". In: International Conference on Space Optics-ICSO 2020. Vol. 11852. International Society for Optics and Photonics. 2021, 118525J.

[27] Hasan Iqbal and Walter O Krawec. "HighDimensional Semiquantum Cryptography". In: IEEE Transactions on Quantum Engineering 1 (2020), pp. 1-17.

[28] Zheshen Zhang et al. "Unconditional security of time-energy entanglement quantum key distribution using dual-basis interferometry". In: Physical review letters 112.12 (2014), p. 120506.

[29] Thomas Brougham et al. "Security of highdimensional quantum key distribution protocols using Franson interferometers". In: Journal of Physics B: Atomic, Molecular and Optical Physics 46.10 (2013), p. 104010.

[30] Damien Stucki et al. "Fast and simple one-way quantum key distribution". In: Applied Physics Letters 87.19 (2005), p. 194108.

[31] Nicolas Gisin et al. "Towards practical and fast quantum cryptography". In: arXiv preprint quantph/0411022 (2004).

[32] Damien Stucki et al. "High rate, long-distance quantum key distribution over $250 \mathrm{~km}$ of ultra low loss fibres". In: New Journal of Physics 11.7 (2009), p. 075003 .

[33] Damien Stucki et al. "Continuous high speed coherent one-way quantum key distribution". In: $\mathrm{Op}$ tics express 17.16 (2009), pp. 13326-13334.

[34] Nino Walenta et al. "A fast and versatile quantum key distribution system with hardware key distillation and wavelength multiplexing". In: New Journal of Physics 16.1 (2014), p. 013047. 
[35] Philip Sibson et al. "Chip-based quantum key distribution". In: Nature communications 8.1 (2017), pp. 1-6.

[36] George L Roberts et al. "Modulator-Free CoherentOne-Way Quantum Key Distribution". In: Laser 8 Photonics Reviews 11.4 (2017), p. 1700067.

[37] Philip Sibson et al. "Integrated silicon photonics for high-speed quantum key distribution". In: $O p$ tica 4.2 (2017), pp. 172-177.

[38] Jincheng Dai et al. "Pass-block architecture for distributed-phase-reference quantum key distribution using silicon photonics". In: Optics Letters 45.7 (2020), pp. 2014-2017.

[39] Cyril Branciard, Nicolas Gisin, and Valerio Scarani. "Upper bounds for the security of two distributed-phase reference protocols of quantum cryptography". In: New Journal of Physics 10.1 (2008), p. 013031.

[40] Tobias Moroder et al. "Security of distributedphase-reference quantum key distribution". In: Physical review letters 109.26 (2012), p. 260501.

[41] Róbert Trényi and Marcos Curty. "Zero-error attack against coherent-one-way quantum key distribution". In: New Journal of Physics 23.9 (2021), p. 093005.

[42] Marcos Curty. "Foiling zero-error attacks against coherent-one-way quantum key distribution". In: Physical Review A 104.6 (2021), p. 062417.
[43] Cyril Branciard et al. "Zero-error attacks and detection statistics in the coherent one-way protocol for quantum cryptography". In: arXiv preprint quant-ph/0609090 (2006).

[44] Maciej Lipiński et al. "White rabbit: A PTP application for robust sub-nanosecond synchronization". In: 2011 IEEE International Symposium on Precision Clock Synchronization for Measurement, Control and Communication. IEEE. 2011, pp. 2530.

[45] Feihu Xu et al. "Secure quantum key distribution with realistic devices". In: Reviews of Modern Physics 92.2 (2020), p. 025002.

[46] Mohammad Mirhosseini et al. "High-dimensional quantum cryptography with twisted light". In: New Journal of Physics 17.3 (2015), p. 033033.

[47] G Cañas et al. "High-dimensional decoy-state quantum key distribution over multicore telecommunication fibers". In: Physical Review A 96.2 (2017), p. 022317.

[48] Frédéric Bouchard et al. "Experimental investigation of high-dimensional quantum key distribution protocols with twisted photons". In: Quantum 2 (2018), p. 111.

[49] Frédéric Bouchard et al. "Quantum process tomography of a high-dimensional quantum communication channel". In: Quantum 3 (2019), p. 138. 


\section{Supplementary Information}

\subsection{Detailed security analysis}

In this part we will revisit the analysis done for Eve's extractable information in more detail.

\subsubsection{Eve's action and constrains}

As a reminder, we can look at Eve's action as a linear transformation 39

$$
\begin{gathered}
|0\rangle_{A}|\varepsilon\rangle_{E} \rightarrow|0\rangle_{B}\left|v_{0}\right\rangle_{E}+\sqrt{Q \mu t}|1\rangle_{B}\left|p_{0}\right\rangle_{E} \\
|\sqrt{\mu}\rangle_{A}|\varepsilon\rangle_{E} \rightarrow|0\rangle_{B}\left|v_{\mu}\right\rangle_{E}+\sqrt{(1-(d-1) Q) \mu t}|1\rangle_{B}\left|p_{\mu}\right\rangle_{E}
\end{gathered}
$$

Where $\mathrm{Q}$ is the quantum bit error rate (QBER) i.e. the probability that Bob accepts the wrong bit value. $\mu$ and $t$ are the pulse occupation and the link transmission. These amplitudes of the states are chosen such that Eve does not change the QBER of the data line.

The loss of coherence is monitored by analyzing the detection events in the monitoring line. The phase between the two arms of the interferometer in the monitoring line is chosen such that two consecutive non-empty pulses sent by Alice will interfere destructively in one output port and constructively in the other port. We label the probability to detect a photon at the constructive port by $P\left(D_{c}\right)$ and the probability to detect a photon at the destructive port by $P\left(D_{d}\right)$. The loss of coherence by Eve's attack is measured by the visibility:

$$
V=\frac{P\left(D_{c}\right)-P\left(D_{d}\right)}{P\left(D_{c}\right)+P\left(D_{d}\right)}
$$

Assuming $\mu t \ll 1$ we can neglect two-photon terms. Eve's action on a consecutive pair of occupied pulses $|\sqrt{\mu}\rangle|\sqrt{\mu}\rangle$ is then given by:

$$
|\sqrt{\mu}, \sqrt{\mu}\rangle_{A}|\varepsilon, \varepsilon\rangle_{E} \rightarrow|0,0\rangle_{B}\left|v_{\mu}, v_{\mu}\right\rangle_{E}+\sqrt{(1-(d-1) Q) \mu t}\left[|1,0\rangle_{B}\left|p_{\mu}, v_{\mu}\right\rangle_{E}+|0,1\rangle_{B}\left|v_{\mu}, p_{\mu}\right\rangle_{E}\right]
$$

The action of the interferometer at Bob's end yields:

$$
|1,0\rangle_{B}\left|p_{\mu}, v_{\mu}\right\rangle_{E}+|0,1\rangle_{B}\left|v_{\mu}, p_{\mu}\right\rangle_{E} \rightarrow\left|D_{c}\right\rangle_{B}\left(\left|p_{\mu}, v_{\mu}\right\rangle_{E}+\left|v_{\mu}, p_{\mu}\right\rangle_{E}\right)+\left|D_{d}\right\rangle_{B}\left(\left|p_{\mu}, v_{\mu}\right\rangle_{E}-\left|v_{\mu}, p_{\mu}\right\rangle_{E}\right)
$$

where we define the constructive and destructive output modes of the interferometer by $\left|D_{c / d}\right\rangle_{B}=\frac{1}{2}\left(|1,0\rangle_{B} \pm\right.$ $\left.|0,1\rangle_{B}\right)$. The probability that the photon is detected at the constructive/destructive detector is thus given by $P\left(D_{c / d}\right) \propto\left|\left(\left|p_{\mu}, v_{\mu}\right\rangle_{E} \pm\left|v_{\mu}, p_{\mu}\right\rangle_{E}\right)\right|^{2}$. The visibility constraint on Eve's action is therefore given by:

$$
V=\left|\left\langle p_{\mu} \mid v_{\mu}\right\rangle\right|^{2}
$$

The third constraint on Eve's transformation is that it must be unitary. In the $\mu t \ll 1$ limit we get

$$
\left\langle v_{0} \mid v_{\mu}\right\rangle=e^{-\mu / 2}
$$

To compute the amount of information that can be extracted by Eve, quantified by the Holevo information, we first need to analyze her action on a qudit with occupation $\mu^{(i)}$ in the $i^{\text {th }}$ time slot. Neglecting all multiple photon terms, Eve's action can be presented by:

$$
\begin{gathered}
\left|0, \ldots, 0, \sqrt{\mu}^{(i)}, 0, \ldots, 0\right\rangle_{A}|\varepsilon, \ldots, \varepsilon\rangle_{E} \rightarrow \\
|0, \ldots, 0\rangle_{B} \otimes\left|V_{i}\right\rangle+\sqrt{(1-(d-1) Q) \mu t}\left|0, \ldots, 0,1^{(i)}, 0 \ldots, 0\right\rangle_{B} \otimes\left|C_{i}\right\rangle+\sum_{k=1 \ldots d, k \neq i} \sqrt{Q \mu t}\left|0, \ldots, 0,1^{(k)}, 0 \ldots, 0\right\rangle_{B}\left|W_{i, k}\right\rangle,
\end{gathered}
$$

where $\left|V_{i}\right\rangle=\left|v_{0}, \ldots, v_{0}, v_{\mu}^{(i)}, v_{0}, \ldots, v_{0}\right\rangle_{E}$ is Eve's state representing the case where she sends a vacuum state at time slot $i,\left|C_{i}\right\rangle=\left|v_{0}, \ldots, v_{0}, p_{\mu}^{(i)}, v_{0}, \ldots, v_{0}\right\rangle_{E}$ is Eve's state representing the case where she sends to Bob a photon at the right time slot $i$, and $\left|W_{i, k}\right\rangle=\left|v_{0}, \ldots, v_{0}, p_{0}^{(k)}, v_{0}, \ldots, v_{0}, v_{\mu}^{(i)}, v_{0}, \ldots, v_{0}\right\rangle_{E}$ is Eve's state representing the case where she sends to Bob a photon at the wrong time slot $k$. 
The density matrix of Eve's subsystem, conditioned by the event where Alice sends a pulse at time slot $i$ and Bob detects a photon at some arbitrary time slot, is given by:

$$
\rho_{E}^{A=i}=(1-(d-1) Q)\left|C_{i}\right\rangle\left\langle C_{i}\left|+\sum_{k=1 \ldots d, k \neq i} Q\right| W_{i, k}\right\rangle\left\langle W_{i, k}\right|
$$

The density matrix of Eve's subsystem conditioned by the event where Bob detects a pulse at time slot $i$ and Alice sent the pulse at an arbitrary time slot:

$$
\rho_{E}^{B=i}=(1-(d-1) Q)\left|C_{i}\right\rangle\left\langle C_{i}\left|+\sum_{k=1 \ldots d, k \neq i} Q\right| W_{k, i}\right\rangle\left\langle W_{k, i}\right|
$$

\subsubsection{Eve's Holevo information}

Eve's information is bounded by the Holevo bound on Alice-Eve channel $\left(\chi_{A E}\right)$ and on Bob-Eve channel $\left(\chi_{B} E\right)$. The maximum amount of information Eve can extract is given by $\max \left\{\chi_{B E}, \chi_{A E}\right\}$. We start by analyzing $\chi_{B E}$, and we will then show that $\chi_{B E}>\chi_{A E}$.

As explained in the main text, Eve can choose the $\left|p_{0}\right\rangle_{E}$ state to be orthogonal to all other states. We now use this to simplify Eve's Holevo information. We start by choosing an orthonormal set of states $G^{\prime}$ the span the space that describes Eve's system for a single time bin, where $\left|p_{0}\right\rangle_{E} \in G^{\prime}$ is one of the states in the set. The basis for Eve's system that spans a $d$-dimension qudit is simply the tensor product of this basis, i.e. $\left|v_{1}, \ldots, v_{d}\right\rangle_{E} \in G=$ $\left|v_{1}\right\rangle_{E} \otimes \ldots \otimes\left|v_{d}\right\rangle_{E} \in \prod_{i=1}^{d} G^{\prime}$.

We can now split the space $G$ to d +2 groups: $G_{i}, i=1 \ldots d$ will consist states with $\left|p_{0}\right\rangle_{E}$ in the $i^{\text {th }}$ time bin, and other vectors from $G^{\prime}$ in rest of the time bins. $G_{d+1}$ will consist states without $\left|p_{0}\right\rangle_{E}$ at any time bin, and $G_{d+2}$ will consist states $\left|p_{0}\right\rangle_{E}$ in more than one time bin. We further define $P_{l}=\sum_{|v\rangle \in G_{l}}|v\rangle\langle v|$ and since $\sum P_{l}=I$, we can express $\chi_{B E}$ by:

$$
\begin{gathered}
\chi_{B E}=S\left(\sum_{i=1}^{d} \frac{1}{d} \rho_{E}^{B=i}\right)-\sum_{i=1}^{d} \frac{1}{d} S\left(\rho_{E}^{B=i}\right) \\
=S\left(\sum_{j=1}^{d+2} P_{j} \sum_{i=1 \ldots d} \frac{1}{d} \rho_{E}^{B=i}\right)-\sum_{i=1 \ldots d} \frac{1}{d} S\left(\sum_{j=1}^{d+2} P_{j} \rho_{E}^{B=i}\right) \\
=S\left(\sum_{j=1}^{d+2} P_{j} \sum_{i=1}^{d} \frac{1}{d}\left((1-(d-1) Q)\left|C_{i}\right\rangle\left\langle C_{i}\left|+\sum_{k=1, k \neq i}^{d} Q\right| W_{k, i}\right\rangle\left\langle W_{k, i}\right|\right)\right) \\
-\sum_{i=1}^{d} \frac{1}{d} S\left(\sum_{j=1}^{d+2} P_{j}\left((1-(d-1) Q)\left|C_{i}\right\rangle\left\langle C_{i}\left|+\sum_{k=1, k \neq i}^{d} Q\right| W_{k, i}\right\rangle\left\langle W_{k, i}\right|\right)\right)
\end{gathered}
$$

We can simplify this expression using the fact that the von-Neumann entropy of a block-diagonal matrix equals the sum of the entropies of the blocks along the diagonal. We claim that the matrices that appear in eq.26) are all block-diagonal, by construction of the sets $G_{i}$. To show this, it is enough to prove that ${ }_{E}\left\langle v\left|\rho_{E}^{A=k}\right| u\right\rangle_{E}=0$ for every $k$ and every $|v\rangle_{E} \in G_{i},|u\rangle_{E} \in G_{j}, j \neq i$. Since each density matrix is by itself a sum of density matrices of pure states, we are left with proving that ${ }_{E}\langle v \mid \varphi\rangle\langle\varphi \mid u\rangle_{E}=0$ for every state $|\varphi\rangle_{E}$ describing Eve's system after a qudit was sent by Alice and received by Bob, represented by $\left|C_{i}\right\rangle=\left|v_{0}, \ldots, v_{0}, p_{\mu}^{(i)}, v_{0}, \ldots, v_{0}\right\rangle_{E}, i \in 1 \ldots d$ or $\left|W_{i, k}\right\rangle=$ $\left|v_{0}, \ldots, v_{0}, p_{0}^{(k)}, v_{0}, \ldots, v_{0}, v_{\mu}^{(i)}, v_{0}, \ldots, v_{0}\right\rangle_{E}, i \neq k \in 1 \ldots d$. It is easy to verify by inspection that ${ }_{E}\left\langle v \mid C_{i}\right\rangle\left\langle C_{i} \mid u\right\rangle_{E}=0$ if $|v\rangle_{E}$ or $|u\rangle_{E}$ are not both in $G_{d+1}$. It can also be verified that ${ }_{E}\left\langle v \mid W_{i, k}\right\rangle\left\langle W_{i, k} \mid u\right\rangle_{E}=0$ if $|v\rangle_{E}$ or $|u\rangle_{E}$ are not both in $G_{k}$. In other words, if $|u\rangle_{E}$ and $|v\rangle_{E}$ are contained in different sets, then ${ }_{E}\left\langle v \mid W_{i, k}\right\rangle\left\langle W_{i, k} \mid u\right\rangle_{E}=0$. We can therefore move the sum over $j$ in eq. $(26)$ outside the entropy $S$ :

$$
\begin{gathered}
\chi_{B E}=\sum_{j=1}^{d+2} S\left(P_{j} \sum_{i=1}^{d} \frac{1}{d}\left((1-(d-1) Q)\left|C_{i}\right\rangle\left\langle C_{i}\left|+\sum_{k=1, k \neq i}^{d} Q\right| W_{k, i}\right\rangle\left\langle W_{k, i}\right|\right)\right) \\
-\sum_{i=1}^{d} \frac{1}{d} \sum_{j=1}^{d+2} S\left(P_{j}\left((1-(d-1) Q)\left|C_{i}\right\rangle\left\langle C_{i}\left|+\sum_{k=1, k \neq i}^{d} Q\right| W_{k, i}\right\rangle\left\langle W_{k, i}\right|\right)\right)
\end{gathered}
$$


Since $P_{l}\left|C_{j}\right\rangle=0$ if $l \neq d+1$ and $P_{l}\left|W_{i, k}\right\rangle=0$ if $l \neq k$, out of all the terms in the sum over $j$ in eq.(26) we are left with the terms $P_{d+1}\left|C_{i}\right\rangle=\left|C_{i}\right\rangle$ and $P_{k}\left|W_{k, i}\right\rangle=\left|W_{k, i}\right\rangle$, which greatly simplifies the expression for the Holevo bound:

$$
\begin{aligned}
\chi_{B E} & \left.=S\left(\sum_{i=1}^{d} \frac{(1-(d-1) Q)}{d}\left|C_{i}\right\rangle\left\langle C_{i}\right|\right)\right)+\sum_{k=1}^{d} \frac{1}{d} S\left(\sum_{i=1, i \neq k}^{d} Q\left|W_{k, i}\right\rangle\left\langle W_{k, i}\right|\right) \\
& -\sum_{i=1}^{d} \frac{1}{d} S\left((1-(d-1) Q)\left|C_{i}\right\rangle\left\langle C_{i}\right|\right)-\sum_{i=1}^{d} \frac{1}{d} S\left(\sum_{k=1, k \neq i}^{d} Q\left|W_{k, i}\right\rangle\left\langle W_{k, i}\right|\right)
\end{aligned}
$$

We Define $d-1$ vectors $\left|W_{i}^{\prime}\right\rangle=\left|v_{0}, \ldots, v_{0}, v_{\mu}^{(i)}, v_{0}, \ldots, v_{0}\right\rangle_{E}, i \in 1 \ldots d-1$ such that $\left|W_{i}^{\prime}\right\rangle \otimes\left|p_{0}\right\rangle=\left|W_{i, d}\right\rangle$ and $\left|W_{i, j}\right\rangle$ are equivalent up to reordering the order of the time slots. Since the entropy is additive for independent systems, rearranging the order of the terms in eq. 27$]$, we get:

$$
\begin{gathered}
\chi_{B E}=\sum_{k=1}^{d}\left[S\left(\sum_{i=1}^{d-1} \frac{Q}{d}\left|W_{i}^{\prime}\right\rangle\left\langle W_{i}^{\prime}\right|\right)-\frac{1}{d} S\left(\sum_{i=1}^{d-1} Q\left|W_{i}^{\prime}\right\rangle\left\langle W_{i}^{\prime}\right|\right)\right] \\
+S\left(\sum_{i=1}^{d} \frac{1-(d-1) Q}{d}\left|C_{i}\right\rangle\left\langle C_{i}\right|\right)-\frac{1}{d} \sum_{i=1}^{d} S\left((1-(d-1) Q)\left|C_{i}\right\rangle\left\langle C_{i}\right|\right)
\end{gathered}
$$

Repeating the above steps from the Holevo bound on the channel between Alice and Eve yields:

$$
\begin{gathered}
\chi_{A E}=\sum_{k=1}^{d}\left[S\left(\sum_{i=1}^{d-1} \frac{Q}{d}\left|W_{i}^{\prime}\right\rangle\left\langle W_{i}^{\prime}\right|\right)-\frac{1}{d} \sum_{i=1}^{d-1} S\left(Q\left|W_{i}^{\prime}\right\rangle\left\langle W_{i}^{\prime}\right|\right)\right] \\
+S\left(\sum_{i=1}^{d-1} \frac{1-(d-1) Q}{d}\left|C_{i}\right\rangle\left\langle C_{i}\right|\right)-\frac{1}{d} \sum_{i=1}^{d} S\left((1-(d-1) Q)\left|C_{i}\right\rangle\left\langle C_{i}\right|\right)
\end{gathered}
$$

To show that the Holevo of the channel between Eve and Bob is higher than the Holveo of Eve and Alics, we notice that $\chi_{B E}-\chi_{A E}=\sum_{i=1}^{d-1} S\left(Q\left|W_{i}^{\prime}\right\rangle\left\langle W_{i}^{\prime}\right|\right)-S\left(\sum_{i=1}^{d-1} Q\left|W_{i}^{\prime}\right\rangle\left\langle W_{i}^{\prime}\right|\right)=(d-1) S(Q)-S\left(\sum_{i=1}^{d-1} Q\left|W_{i}^{\prime}\right\rangle\left\langle W_{i}^{\prime}\right|\right)$. The dimension of the matrix in the second term is at most $d-1$ and thus it cannot have more than $d-1$ nonzero eigenvalues. The maximal entropy of the second term is achieved when all of the eigenvalues are equal, and since its trace is $(d-1) Q$, we conclude that $\sum_{i=1}^{d-1} Q\left|W_{i}^{\prime}\right\rangle\left\langle W_{i}^{\prime}\right| \leq(d-1) S(Q)$. This can also be seen intuitively, as Eve's information over Alice or Bob's state is the same when the correct qudit was transferred, but when an error was passed Eve knows for sure what Bob got but has only partial certainty over what Alice sent. This yields that the Holevo-information will be maximal with Bob.

To calculate the entropy of the above matrices we need to find their eigenvalues. We notice that both $\left|W_{i}^{\prime}\right\rangle$ and $\left|C_{i}\right\rangle$ have the same form, $|u, \ldots u, v, u, \ldots, u\rangle$ for some dimension $(d$ or $d-1)$. We now find in general the eigenvalues of a matrix $M=\sum_{i=1 \ldots n} \overbrace{\left|u, \ldots u, v^{(i)}, u, \ldots, u\right\rangle}^{n \text { terms }}\left\langle u, \ldots u, v^{(i)}, u, \ldots, u\right|$. We can view $|v\rangle$ as $|v\rangle=\alpha|u\rangle+$ $\beta\left|u^{\perp}\right\rangle$ s.t. $\left\langle u \mid u^{\perp}\right\rangle=0$ and $\left|u^{\perp}\right\rangle$ is a unit vector, which gives us $|\langle v \mid v\rangle|^{2}=|\alpha|^{2}+|\beta|^{2}=1$ and $\alpha=\langle u \mid v\rangle$. Now we can define the vectors $|U\rangle=|u, \ldots u\rangle$ and $\left|V_{i}\right\rangle=\left|u, \ldots u, u^{\perp(i)}, u, \ldots, u\right\rangle$ all orthogonal to each other, and get from linearity $M=\sum_{i=1 \ldots n}\left(\alpha|U\rangle+\beta\left|V_{i}\right\rangle\right)\left(\alpha^{*}\left\langle U\left|+\beta^{*}\left\langle V_{i}\right|\right)=n|\alpha|^{2} \mid U\right\rangle\langle U|+\sum_{i=1 \ldots n}\left(\alpha \beta^{*}|U\rangle\left\langle V_{i}\left|+\alpha^{*} \beta\right| V_{i}\right\rangle\left\langle\left. U|+| \beta\right|^{2} \mid V_{i}\right\rangle\left\langle V_{i}\right|\right)\right.$. By narrowing the matrix to the space spanned by $|U\rangle$ and $\left|V_{i}\right\rangle$, we get:

$$
M=\left[\begin{array}{ccccc}
n|\alpha|^{2} & \alpha \beta^{*} & \alpha \beta^{*} & \cdots & \alpha \beta^{*} \\
\alpha^{*} \beta & |\beta|^{2} & 0 & \cdots & 0 \\
\alpha^{*} \beta & 0 & |\beta|^{2} & \cdots & 0 \\
\vdots & \vdots & \vdots & \ddots & \vdots \\
\alpha^{*} \beta & 0 & \cdots & 0 & |\beta|^{2}
\end{array}\right]=|\beta|^{2} I+\left[\begin{array}{cccc}
n|\alpha|^{2}-|\beta|^{2} & \alpha \beta^{*} & \cdots & \alpha \beta^{*} \\
\alpha^{*} \beta & 0 & \cdots & 0 \\
\vdots & & \ddots & \vdots \\
\alpha^{*} \beta & 0 & \cdots & 0
\end{array}\right]=|\beta|^{2} I+M^{\prime}
$$

To obtain the eigenvalues $M$ we can find the roots of the characteristic polynomial of $M^{\prime}$ and add $|\beta|^{2}$ to all of them. $\left|\lambda I-M^{\prime}\right|=\left(\lambda-n|\alpha|^{2}+|\beta|^{2}\right) \lambda^{n}-n|\alpha|^{2}|\beta|^{2} \lambda^{n-1}=\lambda^{n-1}\left(\lambda+|\beta|^{2}\right)\left(\lambda-n|\alpha|^{2}\right)$, so the eigenvalues of $M^{\prime}$ are $n|\alpha|^{2},-|\beta|^{2}$, and 0 with multiplicity $n-1$. Therefore the eigenvalues of $M$ are $n|\alpha|^{2}+|\beta|^{2}$, 0 , and $|\beta|^{2}$ with multiplicity $n-1$, where the zero eigenvalue does not affect the entropy. 
Substituting the above eigenvalues into the expression for Holevo bound on Bob-Eve channel eq.28) information yields:

$$
\begin{gathered}
\chi_{B E}=\sum_{k=1}^{d}\left[\left((d-2) S\left(\frac{Q}{d}\left(1-\left|\left\langle v_{0} \mid v_{\mu}\right\rangle\right|^{2}\right)\right)\right)+S\left(\frac{Q}{d}\left(1+(d-2)\left|\left\langle v_{0} \mid v_{\mu}\right\rangle\right|^{2}\right)\right)\right. \\
\left.-\frac{d-2}{d}\left(S\left(Q\left(1-\left|\left\langle v_{0} \mid v_{\mu}\right\rangle\right|^{2}\right)\right)\right)-\frac{1}{d} S\left(Q\left(1+(d-2)\left|\left\langle v_{0} \mid v_{\mu}\right\rangle\right|^{2}\right)\right)\right] \\
+(d-1) S\left(\frac{1-(d-1) Q}{d}\left(1-\left|\left\langle v_{0} \mid p_{\mu}\right\rangle\right|^{2}\right)\right)+S\left(\frac{1-(d-1) Q}{d}\left(1+(d-1)\left|\left\langle v_{0} \mid p_{\mu}\right\rangle\right|^{2}\right)\right)-\frac{1}{d} \sum_{i=1}^{d} S((1-(d-1) Q))
\end{gathered}
$$

Imposing the unitary constraint for $\left\langle v_{0} \mid v_{\mu}\right\rangle=e^{\mu / 2}$, and noticing that the first line in eq. 31 can be simplified by $S\left(\frac{1}{d} A\right)-\frac{1}{d} S(A)=\sum-\frac{\lambda_{i}}{d} \log _{2}\left(\frac{\lambda_{i}}{d}\right)-\frac{1}{d} \sum-\lambda_{i} \log _{2}\left(\lambda_{i}\right)=S\left(\frac{1}{d}\right) \sum \lambda_{i}$, yields the following expression for the Holevo bound:

$$
\begin{gathered}
\chi_{B E}=Q(d-1) \log _{2}(d) \\
+S\left(\frac{1-(d-1) Q}{d}\left((d-1)\left|\left\langle v_{0} \mid p_{\mu}\right\rangle\right|^{2}+1\right)\right)+(d-1) S\left(\frac{1-(d-1) Q}{d}\left(1-\left|\left\langle v_{0} \mid p_{\mu}\right\rangle\right|^{2}\right)\right)-S(1-(d-1) Q)
\end{gathered}
$$

To maimize $\chi_{B E}$ it is enough to minimize $\left\langle v_{0} \mid p_{\mu}\right\rangle$ under the constraints of eq. 20) and eq. 21). The optimization can be done analytically using a parametric representation of $\left|v_{0}\right\rangle,\left|p_{u}\right\rangle,\left|v_{u}\right\rangle$ in 3-D space [39. This yields:

$$
\left\langle v_{0} \mid p_{\mu}\right\rangle=e^{-\mu / 2} \sqrt{V}-\sqrt{1-e^{-\mu}} \sqrt{1-V}
$$

Equations 32 and 33 provide the maximal entropy Eve can extract from the system as function of the QBER $Q$, the visibility $V$, the occupation $\mu$ and dimension size $d$.

\subsection{Detailed photon detection rate analysis}

One of the caveats of our $d$-dimensional qudits is that it extends each qudit over $d$ time bins. In practice, the expectation time per received qudit is limited by the deadtime of the detector. To calculate the received bit rate as a function of the deadtime of the detectors, we write the expectation value for the number of detection events in time window $\Delta t$ as $\operatorname{clicks}(\Delta t)=\alpha \Delta t$, where $\alpha$ is the detection rate. Assuming detection events are uncorrelated, we can express $\operatorname{clicks}(\Delta t)$ by $\operatorname{clicks}(t)=P(\operatorname{click})(1+\operatorname{clicks}(\Delta t-T-\tau))+(1-P($ click $)) \operatorname{clicks}(\Delta t-\tau)$, where $T$ is the detector deadtime, $\tau$ is the pulse duration and $P($ click $)$ is the probability a qudit will be recorded by the detector. For detector efficiency $\xi$, dimension $d$ and occupation $\mu, P($ click $)=\frac{\xi \mu}{D}$. We therefore get that

$$
\alpha \Delta t=\frac{\xi \mu}{D}(1+\alpha(\Delta t-T-\tau))+\left(1-\frac{\xi \mu}{D}\right) \alpha(\Delta t-\tau)
$$

from which we can extract the received detection rate $\alpha$ :

$$
\alpha=\frac{\frac{\xi \mu}{D}}{(T+\tau) \frac{\xi \mu}{D}+\tau\left(1-\frac{\xi \mu}{D}\right)}=\frac{1}{T+\tau \frac{D}{\xi \mu}}
$$

